\title{
Synthesis and Characterization of Bioinspired Silver Nanoparticles by Aqueous Leaf Extract of Indigofera cassioides: Evaluation of Antimicrobial and Cytotoxic Activity
}

\author{
R. Senthil Kumar1,*, S. Vinoth Kumar'1, M.K.M. Abdul Lathiff 1, G. Muthuboopathi² \\ ${ }^{1}$ Department of Pharmaceutical Chemistry, Swamy Vivekanandha College of Pharmacy, Tiruchengode - 637 205, Tamilnadu, India. \\ ${ }^{2}$ Department of Pharmaceutical Chemistry, Vivekanandha Pharmacy College for Women, Sankari - 637 303, Tamilnadu, India.
}

\section{ARTICLE DETAILS}

\section{Article history:}

Received 08 April 2019

Accepted 21 April 2019

Available online 06 May 2019

\section{Keywords:}

Indigofera cassioides

Green Synthesis

Silver Nanoparticles

Antimicrobial Activity

\begin{abstract}
A B S T R A C T
The green synthesis of silver nanoparticles (AgNPs) has increasingly promising due to their potential applications in nanomedicine. For the first time, silver nanoparticles (AgNPs) were synthesized from aqueous leaf extract of Indigofera cassioides. The AgNPs were characterized by UV-Vis spectroscopy and the characteristic surface plasmon resonance peak was observed at $414 \mathrm{~nm}$. The Fourier transform infrared spectroscopy (FT-IR) revealed the phytochemicals in the plant extract which assisted bioreduction, capping and stabilizing agent. Morphology of AgNPs was characterized by transmission electron microscopy (TEM) and they were spherical shape with average particle size as $20 \mathrm{~nm}$. The face centered cubic (FCC) crystalline structure of the AGNPs was confirmed by X-ray diffraction. Dynamic light scattering (DLS) and zeta potential analysis revealed that the average hydrodynamic diameter of the AGNPs as $80 \mathrm{~nm}$ and the negative zeta potential value of $-36.6 \mathrm{mV}$ indicates good stability. Antimicrobial studies disclosed that the biosynthesized AgNPs possess strong activity against pathogenic bacteria. The cytotoxicity study is concealed that the AgNPs exhibit selective cytotoxicity against the tested cell lines and a pronounced activity was observed against human breast cancer cells (MCF-7). The present study concludes that for the biosynthesized AgNPs could be used as an antimicrobial and anticancer agent.
\end{abstract}

\section{Introduction}

In recent time green synthesis and characterization of nanoparticles have gained more attention due to their widespread applications particularly in biomedical services, cosmetics, drug gene delivery and health care. Silver is of tremendous choice in the field of biological frameworks, living creatures, and drug among the other metals [1]. Silver has been not just demonstrated as a profitable device for hindering and anticipating bacterial diseases yet additionally they are found to display several biological activities. Hence, silver nanoparticles (AgNPs) got a specific consideration for their positive effect in improving numerous parts of economy, including pharmaceuticals, cosmoceuticals, consumables and agriculture and so forth. Modern advancements in nanotechnology include the inclusion of silver nanoparticles into diverse industrial, medical and household products [2,3].

Green synthetic approach of nanoparticle synthesis reduces hazards to the global efforts and implantation of these supportable procedures should take up the essential standards of green chemistry. Green synthesis is relying on the different process parameters and reaction conditions such as temperature, $\mathrm{pH}$, solvent medium, stirring time and reducing agents etc. for the greater stability, high yield and controlled size and shape morphology. Biogenic synthesis is simply a bioengineered benign route and is viewed as better candidates among the distinctive biological elements as they give eco-friendly, economical, safe and valuable route to the biogenesis of metal NPs for the enormous scale production. Many plants are reported to facilitate the formation of AgNPs and their potential applications [4-6]. The amount of gathering of AgNPs varies with reduction potential of ions and the reducing capacity of the plant extract depends on the occurrence of various polyphenols and other heterocyclic compounds $[7,8]$. Plants are known to harbor a major scope of metabolites that are well likely to be responsible for the biogenic synthesis of metal nanoparticles. As plants potentially eliminate the environmental issues by making the NPs more biocompatible, it is necessary to increase the efficiency of locally available and unexplored plants resources for the green synthesis of AgNPs and clarify the possible mechanism involved in synthesis, is still infancy. The Indian medicinal plant, Indigofera cassioides belonging to the family Fabaceae have exhibited excellent pharmacological activities such as antioxidant and free radical scavenging activities, anticancer, chemopreventive, anti-inflammatory and antinociceptive activities [9-11]. By keeping the above contemplations, the present study aimed to biosynthesize silver nanoparticles by a green synthetic methodology, using an aqueous extract derived from Indigofera cassioides leaves. The synthesized AgNPs were comprehensively characterized by different analytical techniques viz. UV-Vis spectroscopy, Infra-red spectroscopy, Powder X-Ray diffraction analysis, dynamic light scattering (DLS), Zeta potential analysis and Transmission Electron Microscopy. Further, the bioengineered AgNPs were oppressed for antimicrobial activities against human pathogenic microorganisms. In vitro cytotoxic properties of AgNPs were additionally evaluated in the present work.

\section{Experimental Methods}

\subsection{Chemicals and Reagents}

Silver nitrate $\left(\mathrm{AgNO}_{3}\right), 3-(4,5$-dimethyl thiazol-2-yl)-5-diphenyl tetrazolium bromide (MTT), fetal bovine serum (FBS), phosphate buffered saline (PBS), Dulbecco's modified Eagle's medium (DMEM) and trypsin were obtained from Sigma Aldrich Co, St Louis, USA. EDTA, glucose and antibiotics from Hi-Media Laboratories Ltd., Mumbai. Dimethyl sulfoxide (DMSO) and propanol from E.Merck Ltd., Mumbai, India. De-ionized water was used throughout the study.

\subsection{Collection and Extraction}

Leaves of Indigofera cassioides were collected from Yercaud hills, Salem in the month of November 2017. The plant was then authenticated by the Joint Director, Botanical Survey of India, Coimbatore, India and a voucher specimen is preserved in our laboratory for reference. The shade dried coarse powder of leaves of I. cassioides $(10 \mathrm{~g})$ was heated with $100 \mathrm{~mL}$ of 
de-ionized water at $60^{\circ} \mathrm{C}$ for 30 minutes. The aqueous extract was filtered through Whatman filter paper No.1, centrifuged at $5000 \mathrm{rpm}$ to get rid of any particulate matters and stored at $4{ }^{\circ} \mathrm{C}$ until further studies.

\subsection{Phytochemical Analysis}

Aqueous leaf extract of $I$. cassioides was subjected to phytochemical tests for the identification active phytoconstituents like carbohydrates, glycosides, tannins, saponins, flavonoids, phenolics, proteins and amino acids as per the standard procedures $[12,13]$.

\subsection{Green synthesis of AgNPs}

The procedure for green production of silver nanoparticles was optimized by changing different physical parameters viz. reaction temperature (at room temperature, $32 \pm 3{ }^{\circ} \mathrm{C}, 50^{\circ} \mathrm{C}$ and $80{ }^{\circ} \mathrm{C}$ ), ratio of extract and silver nitrate solution (1:1, 1:2, 1:3, 1:4 and 1:5) and reaction time (5, 10, 20 and $30 \mathrm{~min}$.). In a typical synthesis of silver nanoparticles, $10 \mathrm{~mL}$ of plant extract was mixed to $50 \mathrm{~mL}$ of $1 \mathrm{mM}$ silver nitrate $\left(\mathrm{AgNO}_{3}\right)$ aqueous solution (1:5 ratio) and the mixture was kept at room temperature with constant stirring on a magnetic stirrer for $30 \mathrm{~min}$. The suspension produced was centrifuged at $3000 \mathrm{rpm}$ for $5 \mathrm{~min}$ and the supernatant liquid was collected. Then the collected supernatant was centrifuged at $15000 \mathrm{rpm}$ for $15 \mathrm{~min}$ and the residue was repeatedly washed with $10 \mathrm{~mL}$ of de-ionized water. Centrifugation-decantationwashing processes were repeatedly done six times to remove impurities if any on the surface of the silver nanoparticles. The obtained nanosuspension was slowly evaporated and dried in an oven at $50{ }^{\circ} \mathrm{C}$ for $24 \mathrm{~h}$. The synthesized silver nanoparticles were then kept for further characterization and antimicrobial studies.

\subsection{Characterization}

Eltek magnetic stirrer was used to synthesize silver nonparticulate. REMI Ultracentrifuge adjusted to $15,000 \mathrm{rpm}$ was used to separate and wash the synthesized nanoparticles. Recording UV-Vis absorption spectra, Shimadzu 1800 spectrophotometer was used. The FT-IR spectrum was recorded using Shimadzu IR Affinity $1 S$ instrument. The X-Ray diffraction (XRD) patterns were recorded using PANalytical (PW3040/60X'/per PRO) $\mathrm{X}$-Ray Diffractometer. Transmission electron microscopy (TEM) was recorded using Philips (CM200) transmission electron microscope. Size distribution and zeta potential of the bioengineered silver nanoparticles in deionized water was measured using dynamic light scattering (DLS) and mixed model measurement phase analysis light scattering in a Zetasizer Nano-ZS, model ZEN3600 equipped with $4.0 \mathrm{~mW}, 633 \mathrm{~nm}$ laser (Malvern Instruments Ltd.).

\subsection{Stability Studies}

Stability of the bioengineered silver nanosuspension in demonized water stored at room temperature was observed by recording UV-Vis spectra at different time intervals viz. day 0, 15, 30 and $60 \mathrm{~min}$.

\subsection{Antimicrobial Study}

Staphylococcus aureus (NCIM-5345), Streptococcus thermophilus (NCIM-2412), Bacillus subtilis (NCIM-5688), Enterococcus faecalis (NCIM5253), Escherichia coli (NCIM-5346), Klebsiella pneumoniae (NCIM-5664), Proteus vulgaris (NCIM-5266), Salmonella typhimurium (NCIM-5278), Candida albicans (NCIM-3628) and Cryptococcus neoformans (NCIM-3541) were procured from National Collection of Industrial Microorganism (NCIM), National Chemical Laboratory, Pune, India. Susceptibility of microorganisms to green synthesized AgNPs and standards was studied using the Kirby-Bauer disc diffusion method in Muller Hinton agar media [14]. The synthesized AgNPs were suspended separately in DMSO to give $1 \mathrm{mg} / \mathrm{mL}$ concentration. From this $100 \mu \mathrm{L}$ was impregnated in blank sterilized discs which contain $100 \mu \mathrm{g} /$ disc. The prepared sample impregnated discs, commercially available discs of standard antibiotics (Cefpodoxime $10 \mu \mathrm{g} /$ disc for bacteria and Fluconazole $25 \mu \mathrm{g} /$ disc for fungi) and a sterile disc contains $100 \mu \mathrm{L}$ of solvent (negative control) were placed carefully on the plates with flamed forceps at least $25 \mathrm{~mm}$ away from the edge. The plates were incubated at $37{ }^{\circ} \mathrm{C}$ for $18-24 \mathrm{~h}$. The diameter of the zones of inhibition was calculated at the end of incubation period. The assay was repetitive thrice and mean was recorded [15].

\subsubsection{Determination of Relative Percentage Inhibition (RPI)}

The relative percentage inhibition of the test compounds with respect to positive controls (Cefpodoxime and Fluconazole) was calculated by the formula, Relative Percentage Inhibition $=(x-y) /(z-y) x 100$, where, $x$ : total area of inhibition of the test compound; $y$ : total area of inhibition of the solvent; z: total area of inhibition of the standard drug. The total area of inhibition was calculated by using an area $=\pi r^{2}$; where $r=$ radius of a zone of inhibition [16].

\subsubsection{Determination of Minimum Inhibitory Concentration (MIC), Minimum}

Bactericidal Concentration (MBC) and Minimum Fungicidal Concentration (MFC)

Minimum inhibitory concentration of synthesized AgNPs was determined by the broth dilution method. In this method, a two-fold serial dilution of biosynthesized AgNPs were prepared in sterile Mueller-Hinton broth to attain a diminishing concentration ranging from $100,50,20,10$, 5 , and $1 \mu \mathrm{g} / \mathrm{mL}$ in six sterile tubes and each dilution was mixed with 100 $\mu \mathrm{L}$ of the standardized bacterial or fungal inoculum $(1.5 \times 108 \mathrm{CFU} / \mathrm{mL})$. The culture tubes were incubated at $37{ }^{\circ} \mathrm{C}$ for $18-24 \mathrm{~h}$. A set of tubes contain only seeded broth (i.e. exclusive of test compound) was kept as control. The lowest concentration that did not permit any observable growth when compared with the control was considered as the MIC.

Minimum bactericidal concentration (MBC)/ minimum fungicidal concentration (MFC) is determined from broth dilution minimum inhibitory concentration (MIC) tests by sub-culturing on agar plates that do not include the test agent. After the MIC determination of the AgNPs, aliquots of $50 \mu \mathrm{L}$ from each tube which showed no observable bacterial augmentation were seeded in agar plates were incubated for $24 \mathrm{~h}$ at $37^{\circ} \mathrm{C}$. The MBC/MFC was observed for incidence or nonappearance of bacterial/fungal growth in agar plates both before and after incubation.

\subsection{In Vitro Anticancer Activity}

\subsubsection{Cell Lines and Cell Culture}

Human liver cancer cells (HepG2), Human breast cancer cells (MCF-7), Human epithelial cancer cells (Hep-2) and Human lung cancer cells (A549) and Normal Human Dermal Fibroblasts (NHDF) cell lines were procured obtained from National Centre for Cell Science (NCCS), Pune, India. The cells were cultured in Dulbecco's Modified Eagles Medium (DMEM), supplement with $10 \%$ heat stabilized fetal bovine serum (FBS). The cell lines were maintained at $37^{\circ} \mathrm{C}$ in a $5 \%$ Carbon dioxide atmosphere and the media were regularly changed.

\subsubsection{Cytotoxic Assay by MTT Method}

The cytotoxicity of the biosynthesized AgNPs on malignant cells and normal cell lines was studied by MTT assay as described earlier [9]. The cells $\left(5 \times 10^{3}\right.$ cells/well) were plated in 96 -well plates along with the medium consisting $10 \%$ FBS and incubated for $24 \mathrm{~h}$ under carbon dioxide atmosphere at $37^{\circ} \mathrm{C}$. The medium was removed and replaced with DMEM containing 1\% FBS and the AgNPs $(100,50,20,10,5,2$ and $1 \mu \mathrm{g} / \mathrm{mL})$ in $0.1 \%$ DMSO at ambient temperature for $15 \mathrm{~min}$. After treatment, the plates were additionally incubated for $48 \mathrm{~h}$ and $10 \mu \mathrm{L}$ of MTT $(5 \mathrm{mg} / \mathrm{mL})$ was added to every well and incubated for extra $4 \mathrm{~h}$. Then $100 \mu \mathrm{L}$ of dimethylsulphoxide (DMSO) was added to each well and the absorbance was measured at $570 \mathrm{~nm}$ in an ELISA reader. The absorbance at $570 \mathrm{~nm}$ was also measured for wells without any sample. The cell viability was calculated using the equation: Cell viability $(\%)=\left[A_{570} \mathrm{~nm}\right.$ of treated cells/A570 nm of untreated cells] x 100.

\section{Results and Discussion}

The current study elucidates the biogenesis of AgNPs using aqueous leaf extract of Indigofera cassioides as a resource of reducing as well as capping agent. Since, I. cassioides is phytochemically affluent plant species whose leaves has been reported with high content of phenolic compounds and flavonoids [10], we hypothesized that I. cassioides leaf extract could reduce $\mathrm{Ag}^{+}$to $\mathrm{Ag}^{0}$ and be utilized for the biosynthesis of AgNPs. Pilot studies with aqueous extract of $I$. cassioides confirmed the creation of AgNPs as evident from the change in colour observed (yellow to dark brown) due to reduction of $\mathrm{Ag}^{+}$ions in solution and distinguishing surface plasmon resonance (SPR) peak between $350 \mathrm{~nm}$ to $450 \mathrm{~nm}$.

Preliminary phytochemical screening of the aqueous extract of leaves of Indigofera cassioides shows the existence of various phytochemical constituents. Carbohydrates, glycosides, flavonoids, tannins, phenolics, protein and amino acids and saponins were present in the extract whereas gums, mucilage and terpenoids were absent in the extract. The results clearly demonstrated that the presence of phytochemical constituents in the extract depends on the solubility of phytochemicals in the polar solvent like water. The results are displayed in Table 1. 
Table 1 Preliminary phytochemical studies of aqueous leaf extract of Indigofera cassioides

\begin{tabular}{ll}
\hline Phytochemical constituents & Present/Absent \\
\hline Carbohydrates & Present \\
Phytosterols & Absent \\
Alkaloids & Absent \\
Glycosides & Present \\
Terpenoids & Absent \\
Proteins \& aminoacids & Present \\
Saponins & Present \\
Tannins & Present \\
Phenolic compounds & Present \\
Flavonoids & Present \\
Fixed oils \& Fats & Absent \\
Gums \& Mucilages & Absent \\
\hline
\end{tabular}

For optimum biosynthesis of AgNPs, various synthetic conditions such as the ratio of the extract of the precursor solution $\left(\mathrm{AgNO}_{3}\right)$, reaction temperature and reaction time were assorted and analyzed for their effect on biosynthesis of AgNPs. All over the optimization experiments, the bioreduction and synthesis of AgNPs were observed by recording UV-Vis spectrum. From varying ratios of extract to $\mathrm{AgNO}_{3}$ in the range $1: 1,1: 2$, $1: 3,1: 4$ and $1: 5(\mathrm{v} / \mathrm{v})$, the colour of the reaction mixture tainted from yellow to brown, the absorbance of SPR peak increased with the gradual increase in the ratio. There was no increase in the absorbance was observed at the ratio of 1:5. This ratio was found to be most suitable for optimum biosynthesis of AgNPs. Similarly, the reaction temperature and time was also illustrious to influence the biosynthesis of AgNPs. It was observed that incubation of the reaction mixture at room temperature $\left(32 \pm 3^{\circ} \mathrm{C}\right)$ for $30 \mathrm{~min}$. were optimum conditions and potentially influences the biogenic synthesis and yield (Fig. 1a-c). In our present study, no further increase in the absorbance was observed after $24 \mathrm{~h}$ indicates that the bioreduction was completed within $30 \mathrm{~min}$. Similarly, further increase in temperature reduces the absorbance of the reaction mixture. Based on these observations, AgNPs biosynthesis was conceded out using optimized circumstances such as $1 \mathrm{mM} \mathrm{AgNO}_{3}, 1: 5$ ratio of extract to the precursor solution and incubation at ambient temperature for $30 \mathrm{~min}$.

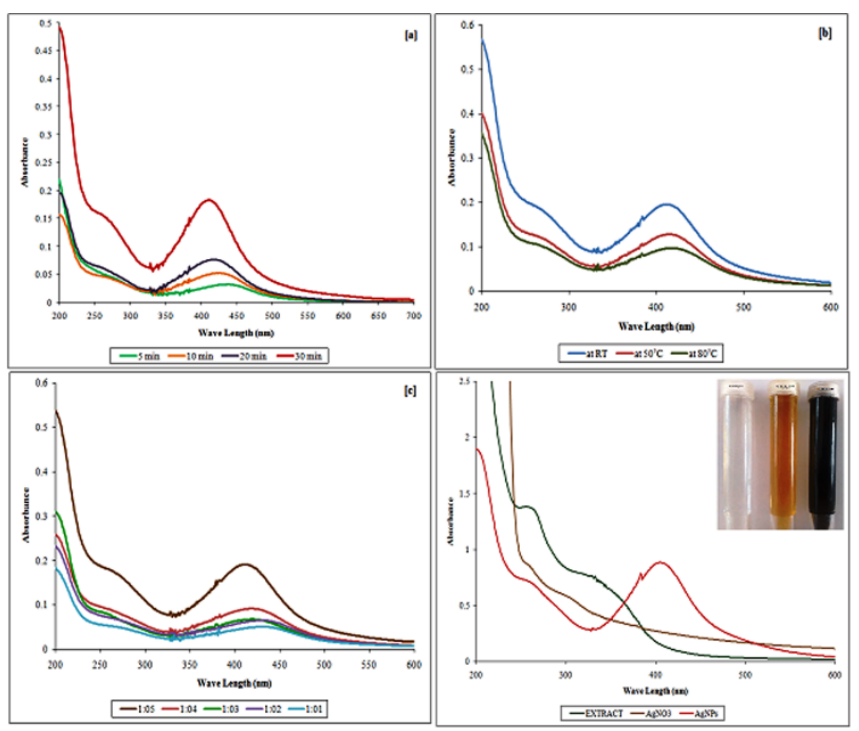

Fig. 1 UV-Visible absorption spectra of biosynthesized AgNPs showing effect of (a) Time (b) Temperature (c) ratios of $\mathrm{AgNO}_{3}$ to extract. The figure with inset shows the UV-Visible spectra of $I$. cassioides leaf extract, $1 \mathrm{mM}$ silver nitrate and biosynthesized AgNPs. The figure in the inset shows the colour of $1 \mathrm{mM}$ silver nitrate, I. cassioides leaf extract and biosynthesized AgNPs

\subsection{UV-Visible Spectral Analysis}

UV-Visible spectroscopy is an essential technique to ascertain the development and stability of biosynthesized nanoparticles. Colloidal solutions and metallic nanoparticles generally appear intensely coloured due to the surface plasmon resonance (SPR) arise from the collective oscillation of free conduction electrons induced by electromagnetic radiations [17]. The development of AgNPs in aqueous solution was established by positioning of the SPR band in the UV-Visible spectral study. Fig. 1 with inset represent the UV-Visible absorption spectrum of aqueous leaf extract of $I$. cassioides, silver nitrate and bioengineered AgNPs. A distinctive peak at $414 \mathrm{~nm}$ was observed for biosynthesized AgNPs, whereas silver nitrate and plant extract did not show any absorption peaks. Shape, size and distribution of nanoparticles were depending upon https://doi.org/10.30799/jnst.222.19050208 the formation of SPR bands. Formation of one SPR band at short wavelengths exposed the presence of tiny sized globular nanoparticles in the reaction mixture [18]. The development of SPR band indicated that the reaction mixture does not have aggregated nanoparticles [19]. Hence a peak at $414 \mathrm{~nm}$ is expected due to the formation of small spherical AgNPs without agglomeration in solution. Absorption bands in the array of 400$420 \mathrm{~nm}$ in the UV-Visible spectrum correspond to round shaped silver nanoparticles. The appearance of a peak at $414 \mathrm{~nm}$ corroborates the sphere-shaped AgNPs according to Mie theory [20].

In addition to small absorption peaks at $258 \mathrm{~nm}$ and $333 \mathrm{~nm}$ was observed in both plant extract and biosynthesized AgNPs. Presence of the peaks might be arising due to the active phytochemicals like amino acids and flavonoids. These bioactive secondary metabolites dynamically interact with $\mathrm{Ag}^{+}$which further led to the development of silver nanoparticles. The bioorganic constituents present in the plant extract facilitate in the charge transfer between bioactive constituents and AgNPs, which get capped over the surface of nanoparticles and provide the stability of the nanoparticles and restricts its aggregation.

\subsection{Fourier Transform Infrared Spectroscopy (FT-IR) Analysis}

FT-IR analysis was conceded out to recognize the biomolecules accountable for the capping and efficient stabilization of biosynthesized AgNPs using aqueous leaf extract of $I$. cassioides as shown in Figs. 2a and b. The FT-IR spectrum of aqueous leaf extract of $I$. cassioides shows strong and broad peak at $3427 \mathrm{~cm}^{-1}$ corresponds to the stretching vibrations of $\mathrm{OH}$ group in alcohols and phenolics compounds and stretching vibrations of $\mathrm{NH}$ in secondary amides. A peak at $2976 \mathrm{~cm}^{-1}$ may be credited to the C$\mathrm{H}$ stretching vibrations. Peaks at $1633 \mathrm{~cm}^{-1}, 1438 \mathrm{~cm}^{-1}$ and $1311 \mathrm{~cm}^{-1}$ denotes stretching vibrations of $\mathrm{CO}$ of carboxylic acids or esters, bending vibration of $\mathrm{N}-\mathrm{H}$ in amides due to the $\mathrm{NH}$ bending to the carbonyl oxygen, stretching vibrations of $\mathrm{N}-\mathrm{H}$ in secondary amine. Peaks at $1242 \mathrm{~cm}^{-1}$ and $1087 \mathrm{~cm}^{-1}$ correspond to the carboxylate group, CO stretching in alcohols, carboxylic acids, esters, ether and ether linkages.

The FT-IR spectrum of AgNPs showed a shift in the place of peaks and intensity distribution. A shift in the peaks was observed at $3427 \mathrm{~cm}^{-1}$ to $3403 \mathrm{~cm}^{-1}$ may be accredited to the reduction of $\mathrm{AgNO}_{3}$. The shift in the absorption frequency indicates the involvement of $-\mathrm{OH}$ group in alcohols and phenols in the reduction process [21]. The peak shift from $1633 \mathrm{~cm}^{-1}$ to $1586 \mathrm{~cm}^{-1}$ and the disappearance of a band at $1438 \mathrm{~cm}^{-1}$ indicates the $\mathrm{N}$ $\mathrm{H}$ group present in the amino acids involved in the bioreduction and stabilization of AgNPs. Peak shift from $1242 \mathrm{~cm}^{-1}$ to $1196 \mathrm{~cm}^{-1}, 1087 \mathrm{~cm}^{-1}$ to $1041 \mathrm{~cm}^{-1}, 810 \mathrm{~cm}^{-1}$ to $793 \mathrm{~cm}^{-1}$ and vanishing of $1298 \mathrm{~cm}^{-1}$ peak in the FT-IR spectrum of AgNPs illustrate the involvement of carboxylic acids and esters in the silver nanoparticles development and stabilization.

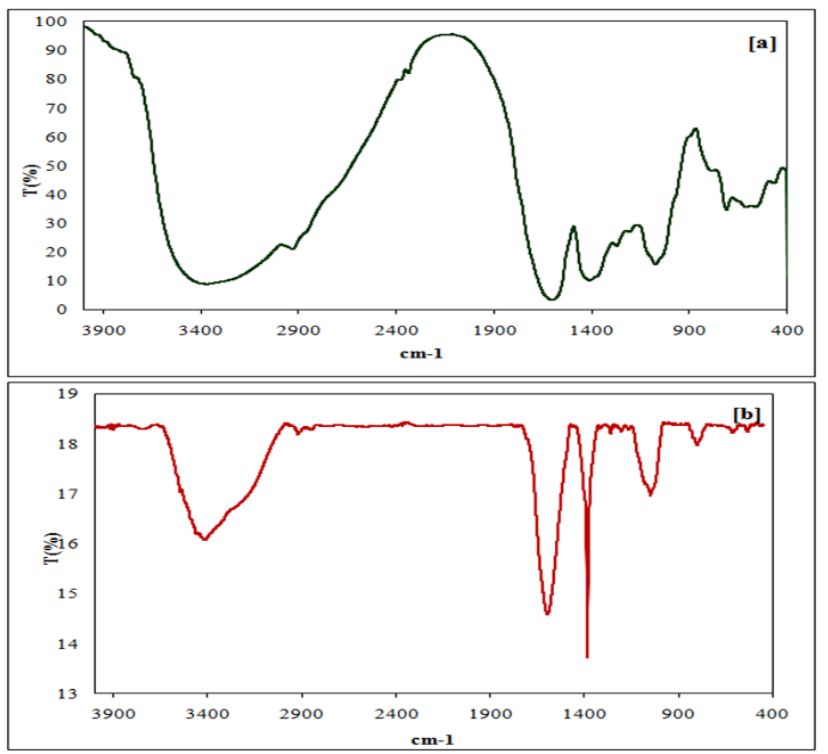

Fig. 2 FT-IR spectra of (a) Aqueous leaf extract of I. cassioides and (b) biosynthesized AgNPs

The FT-IR spectra of the aqueous leaf extract of I. cassioides (Fig. 2a) and bioengineered AgNPs (Fig. 2b) demonstrate change in peak intensities and peak shift indicating the involvement of carboxyl, hydroxyl and amide groups in the reduction and stabilization of AgNPs. FT-IR studies have established that the carboxy group of amino acids and amide group of proteins has tough affinity to bind the silver representing the proteins could most likely form a coating on the surface of AgNPs to thwart agglomeration [22]. Phytochemical compounds like flavonoids, phenolics, 
amino acids, carbohydrates, proteins and ascorbic acid present in the plant extracts concerned in the bioreduction and stabilization of AgNPs and the biosynthesized nanoparticles are environmentally benign and nontoxic [23]. Phytochemical analysis of the plant extract also supports the present findings.

\subsection{X-Ray Diffraction (XRD) Analysis}

The X-ray diffraction pattern of the synthesized AgNPs is displayed in Fig. 3. The diffraction peaks of AgNPs were observed at $2 \theta$ values of $38.22^{\circ}$. $44.36^{\circ}, 64.64^{\circ}, 77.42^{\circ}$ and $81.36^{\circ}$ resultant to lattice plane values indexed at (111), (200), (220), (311) and (222) crystalline planes of the face centered cubic (fcc) crystalline structure of silver respectively. The results observed are in good harmony with the FCC structure from the Joint Committee on Powder Diffraction Standards (JCPDS) file no.: JCDD-PDF-2, Release 2007, PA, USA, 2007 (JCPDS Card No.: 04-0783). Hence, the XRD spectrum evidently indicates that the biosynthesized AgNPs are in crystalline structure. Broadening of peak can be attributing to the development of nanoparticles. Using the Debye-Scherrer's formula, the average crystallite size of biosynthesized AgNPs was calculated.

XRD measures the crystallite size which is consistent diffracting crystalline domain and the estimated average crystallite size of the particle is $18.39 \pm 1.42 \mathrm{~nm}$ (Mean \pm SEM) calculated from the width of (111) Bragg's reflection. Besides, Bragg's peaks representation of silver nanoparticles, additional peaks was also observed at the diffraction angles of $28.03^{\circ}, 32.36^{\circ}, 46.33^{\circ}$ and $54.89^{\circ}$. The peaks present at $28.03^{\circ}$ and $32.36^{\circ}$ obscure the possible existence of $\mathrm{Ag}_{2} \mathrm{O}$ [24]. The other peaks might be due to the involvement of phytochemical moieties presents in the aqueous leaf extract of I. cassioides. Generally, specific phase width is directly proportional to the mean nanoparticles size. In present results, broader peaks point out the involvement of aqueous leaf extract of $I$. cassioides in crystal nuclei growth and particle formation. The peak position and crystallite size of the biosynthesized AgNPs are displayed in Table 2.

Table 2 XRD data of biosynthesized AgNPs from aqueous leaf extract of $I$. cassioides

\begin{tabular}{llll}
\hline Peak position $(2 \theta)$ & FWHM & Miller Indices & Crystallite Size $(\mathrm{nm})$ \\
\hline 38.22 & 12.8248 & $(111)$ & 18.8356 \\
44.36 & 11.5142 & $(200)$ & 17.2541 \\
64.64 & 11.784 & $(220)$ & 19.3511 \\
77.42 & 12.722 & $(311)$ & 22.6237 \\
81.36 & 7.5972 & $(222)$ & 13.9021 \\
Crystallite size range & & & $13-22 \mathrm{~nm}$ \\
Average crystallite size (Mean \pm SEM) & & $18.39 \pm 1.42 \mathrm{~nm}$ \\
\hline
\end{tabular}

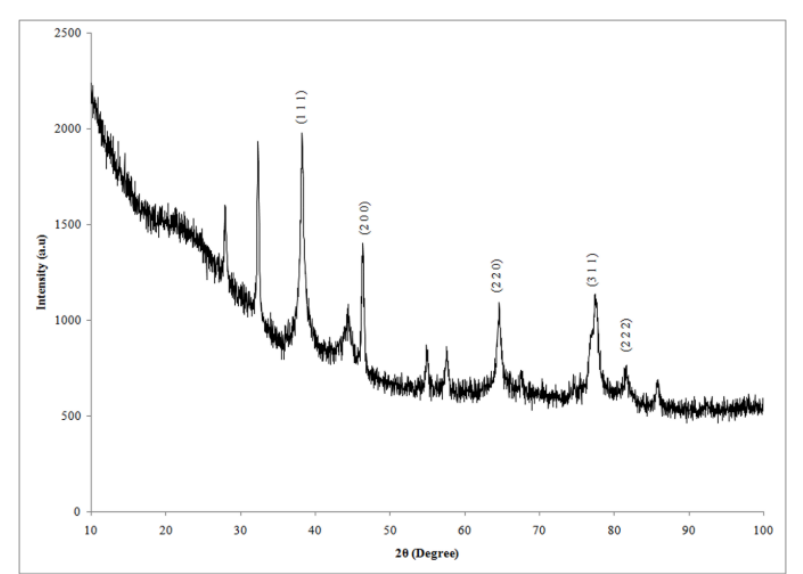

Fig. 3 XRD pattern of biosynthesized AgNPs from aqueous leaf extract of I. cassioides

\subsection{Transmission Electron Microscopy (TEM) Analysis}

Morphology and size of the nanoparticles are essential factors for the production of any metallic nanoparticles. The morphology and magnitude of the biosynthesized silver nanoparticles were examined by TEM analysis. TEM images at diverse magnifications and selected area electron diffraction (SAED) patterns are shown in Fig. 4(a-d). The TEM results illustrate that the biosynthesized AgNPs were mostly spherical in shape with wide variation in size of the particles and the spherical distribution of AgNPs have given rise to symmetric SPR band [25]. The biosynthesized AgNPs are enclosed by a thin coating of capping bioorganic material from the aqueous leaf extract of $I$. cassioides as manifest from the TEM images. The capping bioorganic materials prevent clumping of AgNPs and provide additional stability of the AgNPs. Similar results were reported in biosynthesis of silver nanoparticles from leaf extract of Fraxinus excelsia [21].

https://doi.org/10.30799/jnst.222.19050208
The size of biosynthesized AgNPs ranges from 11 to $30 \mathrm{~nm}$ with an average size of $20.18 \pm 0.3858$ (Mean \pm SEM) which is in good harmony with XRD analysis. Further the morphology of biosynthesized AgNPs was spherical without much aggregate is in excellent agreement with the SPR band shape obtained in the UV-Visible spectra. The particle size distribution is illustrated in Fig. 4(d). Morphology and crystallite nature of biosynthesized AgNPs was again evident from selected area electron diffraction (SAED) pattern of the nanoparticles which is shown in Fig. 4(c). The indexed SAED patterns of the AgNPs with bright spots indicate the crystalline nature of the silver nanoparticles. The ring nearer to the center and is high intensity may be accredited to (111) reflection. The succeeding ring is indexed to the (200) reflection. The third, fourth and fifth ring belongs to (220), (311) and (222) reflections, respectively. The SAED pattern showed fringes with clear, bright circular rings suggested the extremely crystalline nature of the biosynthesized AgNPs. The results endorsed from XRD are in good conformity with the SAED pattern obtained.

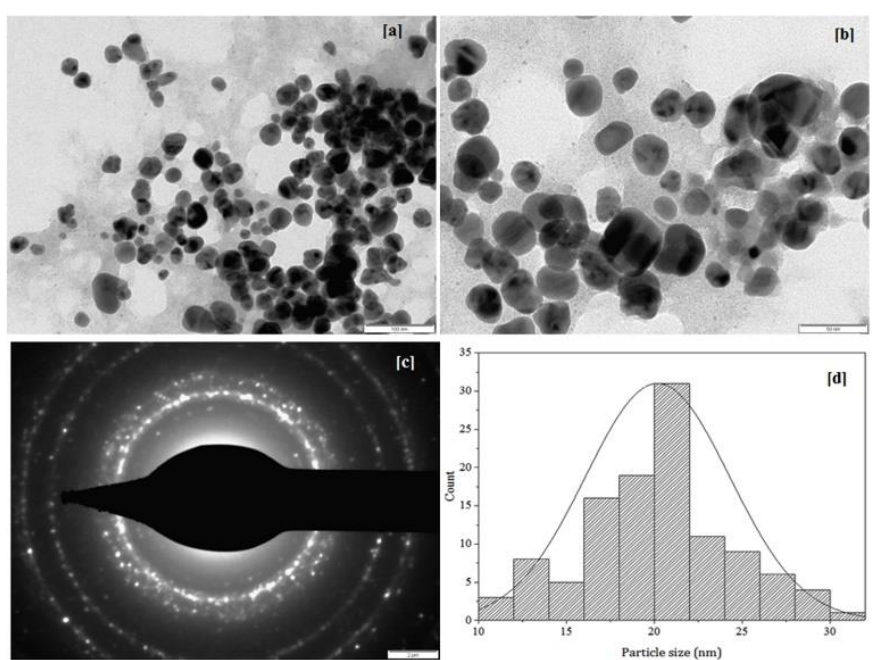

Fig. 4 Transmission electron microscopy (TEM) images of biosynthesized AgNPs with different scale bars (a) $100 \mathrm{~nm}$ (b) $50 \mathrm{~nm}$ (c) SAED pattern and (d) histogram for particle size distribution

\subsection{Dynamic Light Scattering (DLS) and Zeta Potential Analysis}

The distribution of particle size and the zeta potential was measured using the nanoparticles analyzer. Fig. 5(a) showed the mean particle size distribution of biosynthesized AgNPs have a mean particle size of 80.67 $\mathrm{nm}$, which was greater than the average particle size analyzed by TEM. The difference in the diameter of nanoparticles values between TEM and DLS measurement was mostly due to the process concerned in the sample preparation. The particle size resolved by TEM correspond to the actual diameter of the nanoparticles as it was measured at the actual state of the sample, whereas by the dynamic light scattering method (DLS), the hydrodynamic diameter i.e. hydrated state, of the nanoparticles were obtained; therefore, the nanoparticles will have a larger hydrodynamic volume due to the solvent effects in the hydrated state [26].

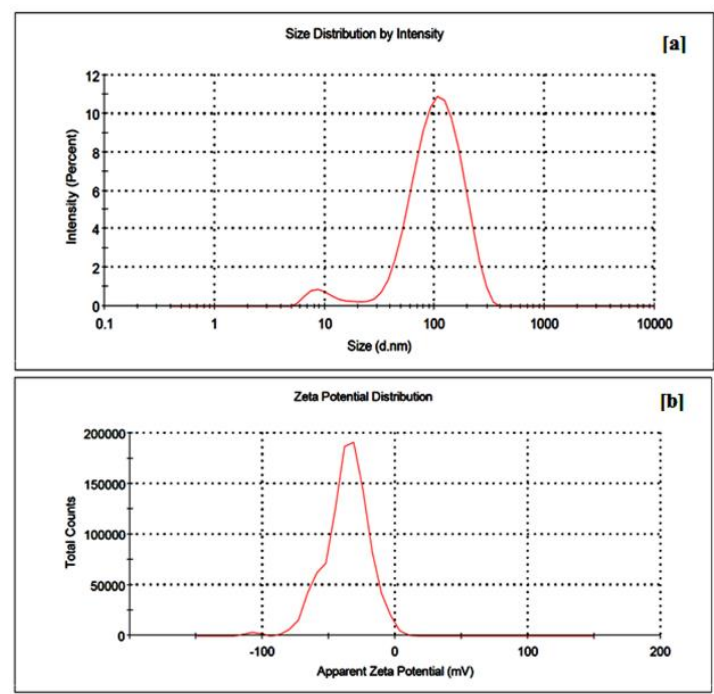

Fig. 5 (a) Particle size distribution (b) Zeta potential distribution of biosynthesized AgNPs by dynamic light scattering (DLS) technique 
Zeta potential is a sign of the stability of nano-suspension. The degree of the zeta potential designates the extent of electrostatic repulsion between adjacent, similarly charged particles in dispersion. Nanosuspensions with high zeta potential (negative or positive) are electrically stabilized which nanosuspensions with low zeta potential be likely to coalesce or flocculate. Zeta potential of the biosynthesized AgNPs was $36.6 \mathrm{mV}$ as showed in the Fig. 5(b). The high negative potential value confirms high dispersity of the AgNPs and also indicates extended stability of the biosynthesized silver nanoparticles. The negative zeta potential value was attributed to the presence of a good capping layer around the nanoparticles formed by the phytochemicals in the aqueous extract of $I$. cassioides responsible for electrostatic stabilization of nano-colloidal solution. The negative charge of biosynthesized AgNPs might be due to the presence of polyphenols present in the extract [27]. The result is in good agreement with previous studies [28,29].

Polydispersity index (PDI) value indicates the range of nanoparticles size distribution. PDI ranges from a value of 0.01 to 0.7 for monodispersed particles. Samples with very broad size distribution have PDI greater than $0.7[30,31]$. The PDI of the biosynthesized AgNPs was found to be 0.269 indicating the biosynthesized nanoparticles are monodispersed with greater stability.

\subsection{Stability Studies}

Stability of nanoparticles is a crucial factor for its long-term use. Stability of the biosynthesized AgNPs was confirmed by the analyses of zeta potential and polydispersity index. However, stability of biosynthesized AgNPs is firmly depends on the degree of aggregation, can be monitored by the change in the SPR band. Stability of biosynthesized AgNPs using aqueous leaf extract of $I$. cassioides were analysed by maintaining the malpractices in de-ionized water at room temperature for 60 days without light protection. The results obtained revealed that the biosynthesized AgNPs were fairly stable without any reasonable aggregation. Fig. 6 depicts this observation quite satisfactory. In this figure, the spectra indicated the SPR bands of plant extract mediated AgNPs solution at day $0,15,30$ and 60 . The solution containing the nanoparticles was reasonably stable even after 60 days though the SPR absorbance have been slightly reduced because of some aggregation of nanoparticles which was observed visually [26]. Analyzing these spectroscopic results, we can propose that the biosynthesized AgNPs using aqueous leaf extract of $I$. cassioides are reasonably stable at room temperature and does not show signs of any significant aggregation.

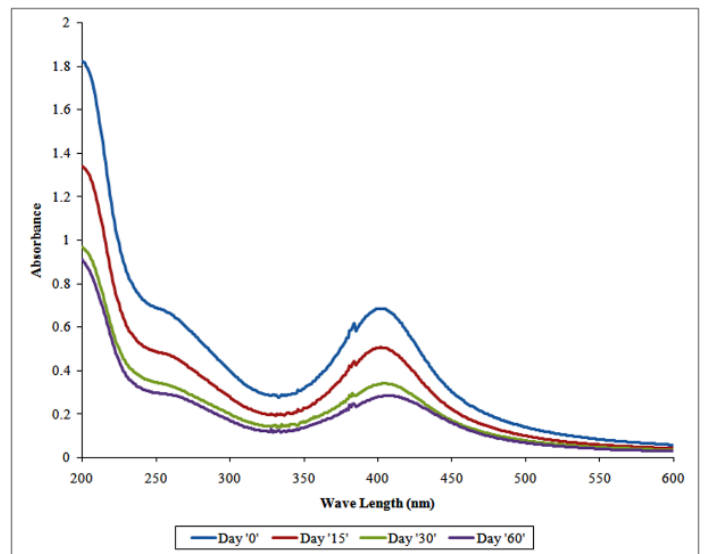

Fig. 6 Stability of biosynthesized AgNPs by successive UV-Visible absorption spectra at different time intervals

\subsection{Antimicrobial Activity of Biosynthesized AgNPs}

The antibacterial activity of synthesized AgNPs were investigated against four gram (+)ve pathogens, namely $S$. aureus, $S$. thermophilus, $E$. faecalis and B. subtilis; four gram (-)ve organisms namely, E. coli, $K$. pneumonia, $P$. vulgaris and $S$. typhimurium; two fungal strains namely $C$. albicans and $C$. neoformans by disc diffusion method at the concentration of $100 \mu \mathrm{g} / \mathrm{mL}$ concentration. Cefpodoxime and fluconazole were used as standard drugs for antibacterial and antifungal screening, respectively. The mean zone of inhibition produced by the aqueous leaf extract and biosynthesized AgNPs are shown in Table 3. The results revealed that the silver nanoparticles displayed significant inhibitory activity against the all the tested microorganisms. The highest value was recorded against $S$. thermophilus and the lowest value was recorded against $S$. aureus among the gram-positive microorganisms. In case of gram-negative pathogens, maximum activity was observed against $E$. coli and the lowest against $P$. vulgaris. Further, the biosynthesized AgNPs were screened against to fungal strains and the result revealed good antifungal activity. Maximum activity was observed against $C$. albicans, whereas moderate activity was observed against $C$. neoformans. Relative percentage inhibition of aqueous leaf extract and biosynthesized AgNPs were calculated. The results obtained are displayed in Table 4. The results revealed that the biosynthesized silver nanoparticles produced better antimicrobial activity against the tested organism and the activity was found to be superior to the plant extract. This indicates antimicrobial activity of silver nanoparticles by releasing silver ions which induce toxicity to the microorganisms. The minimum inhibitory concentration (MIC), minimum bactericidal concentration (MBC) and minimum fungicidal concentration (MFC) were also studies for the aqueous leaf extract and biosynthesized AgNPs by broth dilution technique. The results obtained have been clearly demonstrated that the biosynthesized silver nanoparticles exhibit potent antibacterial and antifungal activity. The results are presented in Table 5.

Table 3 Antimicrobial activity of biosynthesized AgNPs

\begin{tabular}{|c|c|c|c|c|}
\hline \multirow{2}{*}{$\begin{array}{l}\text { Type of } \\
\text { microorganism }\end{array}$} & \multirow{2}{*}{$\begin{array}{l}\text { Name of the } \\
\text { microorganism }\end{array}$} & \multicolumn{3}{|c|}{ Diameter zone of inhibition $(\mathrm{mm})(100 \mu \mathrm{g} / \mathrm{mL})^{\#}$} \\
\hline & & Extract & AgNPs & Std.* \\
\hline \multirow{4}{*}{ Gram (+)ve } & S. aureus & $12.3 \pm 1.08$ & $22.3 \pm 1.15$ & $24.4 \pm 1.83$ \\
\hline & S. thermophilus & $16.4 \pm 1.12$ & $32.2 \pm 1.88$ & $34.3 \pm 2.16$ \\
\hline & E. faecalis & $14.3 \pm 1.02$ & $31.6 \pm 1.04$ & $35.2 \pm 2.87$ \\
\hline & B. subtilis & $17.3 \pm 1.32$ & $24.4 \pm 1.23$ & $26.6 \pm 1.13$ \\
\hline \multirow{4}{*}{ Gram (-)ve } & E. coli & $16.4 \pm 1.13$ & $24.1 \pm 1.87$ & $28.2 \pm 2.11$ \\
\hline & K. pneumoniae & $12.6 \pm 0.89$ & $19.6 \pm 1.12$ & $21.6 \pm 1.87$ \\
\hline & P. vulgaris & $12.4 \pm 1.06$ & $17.4 \pm 1.04$ & $18.2 \pm 1.12$ \\
\hline & S. typhimurium & $15.4 \pm 1.23$ & $23.8 \pm 1.91$ & $24.6 \pm 1.24$ \\
\hline \multirow{2}{*}{ ungi } & C. albicans & $13.4 \pm 0.98$ & $27.4 \pm 1.32$ & $29.1 \pm 2.14$ \\
\hline & C. neoformans & $12.6 \pm 1.14$ & $18.3 \pm 1.37$ & $21.2 \pm 1.68$ \\
\hline
\end{tabular}

Data are expressed as Mean \pm SEM; \#Average of three independent determinations ${ }^{*}$ Cefpodoxime $(10 \mu \mathrm{g} /$ disc) for both Gram (+)ve and Gram (-)ve organism; Fluconazole (25 $\mathrm{\mu g} /$ disc) for fungi.

Table 4 Relative percentage inhibition of biosynthesized AgNPs

\begin{tabular}{llll}
\hline Type of & Name of the & \multicolumn{2}{l}{ Relative Percentage Inhibition } \\
\cline { 2 - 4 } microorganism & microorganism & Extract & AgNPs \\
\hline \multirow{3}{*}{ Gram (+)ve } & S. aureus & 50.41 & 91.39 \\
& S. thermophilus & 47.81 & 93.87 \\
& E. faecalis & 40.62 & 89.77 \\
& B. subtilis & 67.58 & 91.72 \\
\hline \multirow{2}{*}{ Gram (-)ve } & E. coli & 58.16 & 85.46 \\
& K. pneumonia & 58.33 & 90.74 \\
& P. vulgaris & 68.13 & 95.6 \\
Fungi & S. typhimurium & 62.60 & 96.74 \\
\hline & C. albicans & 46.05 & 94.15 \\
& C. neoformans & 59.43 & 86.32 \\
\hline
\end{tabular}

Table 5 Minimum inhibitory concentration (MIC) and minimum bactericidal/ fungicidal concentration (MBC/MFC) of biosynthesized AgNPs

\begin{tabular}{|c|c|c|c|c|c|c|}
\hline \multirow[t]{2}{*}{$\begin{array}{l}\text { Type of } \\
\text { microorganism }\end{array}$} & \multirow[t]{2}{*}{$\begin{array}{l}\text { Name of the } \\
\text { microorganism }\end{array}$} & \multicolumn{2}{|c|}{$\begin{array}{l}\text { MIC } \\
(\mu \mathrm{g} / \mathrm{mL})\end{array}$} & \multicolumn{2}{|c|}{$\begin{array}{l}\text { MBC/MFC } \\
(\mu \mathrm{g} / \mathrm{mL})\end{array}$} & \multirow{2}{*}{$\begin{array}{l}\text { MBC(or) } \\
\text { MFC/MIC } \\
\text { Ratio }\end{array}$} \\
\hline & & Extract & AgNPs & Extract & AgNPs & \\
\hline \multirow{4}{*}{ Gram (+)ve } & S. aureus & 20 & 10 & 50 & 10 & 1.0 \\
\hline & S. thermophilus & 20 & 5 & 50 & 5 & 1.0 \\
\hline & E. faecalis & 20 & 10 & 50 & 20 & 2.0 \\
\hline & B. subtilis & 50 & 2 & 100 & 5 & 2.5 \\
\hline \multirow{4}{*}{ Gram (-)ve } & E. coli & 20 & 5 & 50 & 5 & 1.0 \\
\hline & K. pneumonia & 50 & 5 & 100 & 5 & 1.0 \\
\hline & P. vulgaris & 20 & 5 & 50 & 10 & 2.0 \\
\hline & S. typhimurium & 50 & 5 & 100 & 5 & 1.0 \\
\hline \multirow{2}{*}{ Fungi } & C. albicans & 50 & 10 & 100 & 20 & 2.0 \\
\hline & C. neoformans & 50 & 20 & 100 & 50 & 1.0 \\
\hline
\end{tabular}

\subsection{In Vitro Anticancer Study}

The unique properties of AgNPs are advantageous for cancer chemotherapy since they led to an improved therapeutic efficacy with minimum systemic toxicity. Plant extract mediated biosynthesized AgNPs produced pronounced toxic effect against the cancer cells than normal calls which indicating the target specific toxicity potential of AgNPs [32] Taking into consideration, the in vitro cytotoxicity study was performed against four human cancer cell lines and one normal human cell line namely, Human liver cancer cells (HepG2), Human breast cancer cell line (MCF-7), Human epithelial carcinoma (Hep-2) and Human lung cancer cells (A549) and Normal Human Dermal Fibroblasts (NHDF) by MTT assay method. The results are presented in Table 6. Various concentrations of biosynthesized AgNPs were treated with these cell lines for $48 \mathrm{~h}$. The cell 
viability was concentration dependent with an increase in concentration of AgNPs the viability is decreased. Based on the $\mathrm{IC}_{50}$, the biosynthesized AgNPs produced selective cytotoxicity towards the cancer cells than the normal cell line. This selective cytotoxicity of AgNPs may be due to the induction of oxidative stress and the phytochemicals present in the plant extract.

Table 6 In vitro cytotoxicity of biosynthesized AgNPs

\begin{tabular}{lll}
\hline Cell Lines & \multicolumn{2}{l}{$\mathrm{IC}_{50}$ Values $(\mu \mathrm{g} / \mathrm{mL})^{*}$} \\
\cline { 2 - 3 } & AgNPs & Cisplatin \\
\hline HepG2 (Human liver cancer cells) & $10.20 \pm 1.21$ & $8.12 \pm 0.91$ \\
MCF7 (Human breast adenocarcinoma) & $9.35 \pm 0.809$ & $4.54 \pm 0.34$ \\
A549 (Small cell lung cancer cells) & $12.33 \pm 1.108$ & $7.59 \pm 0.88$ \\
Hep2 (Human epithelioma) & $12.63 \pm 1.092$ & $9.42 \pm 0.89$ \\
NHDF (Normal human dermal fibroblasts) & $67.84 \pm 5.15$ & $87.41 \pm 3.42$ \\
\hline
\end{tabular}

Data are expressed as Mean \pm SEM; ${ }^{*}$ Average of three independent determinations

\section{Conclusion}

Use of aqueous leaf extract of Indigofera cassioides for the synthesis of silver nanoparticles is a novel step towards the biogenic green synthesis of silver nanoparticles. It is an eco-friendly, rapid and non-toxic approach. Further, application of biogenic silver nanoparticles against different human pathogens and cancer cell lines established its efficiency against a wide range of microorganisms and cancer cells. Due to the pharmacological activities of green synthesized silver nanoparticles, it can be used in different formulations like nano-fungicides, nanoantimicrobials and anticancer drug carriers. Lastly, most significant is that it is a cost-efficient approach as raw materials involved in the synthesis are very cheap.

\section{References}

[1] V. Parashar, R. Parashar, B. Sharma, A.C. Pandey, Parthenium leaf extract mediated synthesis of silver nanoparticles, a novel approach towards weed utilization, Dig. J. Nanomater. Biostruct. 4 (2009) 45-50.

[2] W.L. Lee, S. Mahendra, K. Zodrow, D. Li, Y.C. Tsai, et al., Developmental phytotoxicity of metal oxide nanoparticles to Arabidopsis thaliana, Environ. Toxicol. Chem. 29 (2010) 669-675.

[3] E. Navarro, A. Baun, R. Behra, N.B. Hartmann, J. Filser, et al., Environmental behavior and ecotoxicity of engineered nanoparticles to algae, plants, and fungi, Ecotoxicol. 17 (2008) 372-386.

[4] A. Saxena, R.M. Tripathi, R.P. Singh, Biological synthesis of silver nanoparticles by using onion (Allium cepa) extract and their antibacterial activity, Dig. J. Nanomater. Biostruct. 5 (2010) 427-432.

[5] A.I. Waidha, V. Pandiyarasan, T. Anusya, K.M. Waidha, A.H. Shah, Synthesis and characterization of silver nano rod like structures by green synthesis method using Curcumin longa, Int. J. ChemTech Res. 7 (2015) 1504-1508.

[6] D. Arumai Selvan, D. Mahendiran, R. Senthil Kumar, A. Kalilur Rahiman, Garlic, green tea and turmeric extracts-mediated green synthesis of silver nanoparticles: Phytochemical, antioxidant and in vitro cytotoxicity studies, J. Photochem. Photobiol. B 180 (2018) 243-252.

[7] R. Nair, S.H. Varghese, B.G. Nair, T. Maekawa, Y. Yoshida, S.D. Kumar, Nanoparticulate material delivery to plants, Plant Sci. 179 (2010) 154-163.

[8] A. Vedpriya, Living systems eco-friendly nanofactories, Dig. J. Nanomater. Biostruct. 5 (2010) 9-21.

[9] R.S. Kumar, B. Rajkapoor, P. Perumal, In vitro and in vivo anticancer activity of Indigofera cassioides Rottl. Ex. DC., Asian Pac. J. Trop. Med. 4 (2011) 379-485.

[10] R.S. Kumar, B. Rajkapoor, P. Perumal, Antioxidant activities of Indigofera cassioides Rottl. Ex. DC. using various in vitro assay models, Asian Pac. J. Trop. Biomed. 2 (2012) 256-261.
[11] R. Senthil Kumar, B. Rajkapoor, P. Perumal, Anti-inflammatory and antinociceptive activities of methanolic leaf extract of Indigofera cassioides Rottl. Ex. DC., J. Acute Dis. 2 (2013) 322-326.

[12] J.B. Harborne, Methods of plant analysis, Chapman and Hall, London, 1973.

[13] H. Wagner, S. Bladt, E.M. Zgainski, Plant drug analysis, Springer - Verlag, Berlin, 1984

[14] A.W. Bauer, W.M.M. Kirby, J.C. Sherris, M. Turck, Antibiotic susceptibility testing by a standardized single disk method, Am. J. Clin. Pathol. 45 (1966) 493496.

[15] M.R.S. Zaiden, A. Noor Rain, A.R. Badrul, A. Adlin, A. Norazah, I. Zakiah, In vitro screening of five local medicinal plants for antibacterial activity using disc diffusion method, Trop. Biomed. 22 (2005) 165-170.

[16] K. Gaurav Kumar, L. Karthik, K.V. Bhaskara Rao, In vitro anti-candida activity of Calotropis gigantean against clinical isolates of Candida, J. Pharm. Res. 3 (2010) 539-542.

[17] M.A. Noginov, G. Zhu, M. Bahoura, J. Adegoke, C. Small, et al., The effect of gain and absorption on surface plasmons in metal nanoparticles, Appl. Phys. B 86 (2007) 455-460.

[18] R. Kumar, S.M. Roopan, A. Prabhakarn, V.G. Khanna, S. Chakroborty, Agricultural waste Annona squamosa peel extract: biosynthesis of silver nanoparticles, Spectrochim. Acta A: Mol. Biomol. Spectrosc. 90 (2012) 173-176.

[19] M. Moyo, M. Gomba, T. Nharingo, Afzelia quanzensis bark extract for green synthesis of silver nanoparticles and study of their antibacterial activity, Int. J. Ind. Chem. 6 (2015) 329-338.

[20] T.C. Prathna, N. Chandrasekaran, A.M. Raichur, A. Mukherjee, Biomimetic synthesis of silver nanoparticles by Citrus limon (lemon) aqueous extract and theoretical prediction of particle size, Colloids Surf. B Biointerf. 82 (2011) 152159.

[21] M. Parveen, F. Ahmad, A.M. Malla, S. Azaz, Microwave-assisted green synthesis of silver nanoparticles from Fraxinus excelsior leaf extract and its antioxidant assay, Appl. Nanosci. 6 (2016) 267-276.

[22] B. Sadeghi, A. Rostami, S.S Momeni, Facile green synthesis of silver nanoparticles using seed aqueous extract of Pistacia atlantica and its antibacterial activity, Spectrochim. Acta A Mol. Biomol. Spectrosc. 134 (2015) 326-332.

[23] M. Sathishkumar, K. Sneha, Y.S. Yun, Green fabrication of zirconia nano-chains using novel Curcuma longa tuber extract, Mater. Lett. 98 (2013) 242-245.

[24] Q. Sun, X. Cai, J. Li, M. Zheng, Z. Chen, C.P. Yu, Green synthesis of silver nanoparticles using tea leaf extract and evaluation of their stability and antibacterial activity, Colloids Surf. A Physicochem. Eng. Aspec. 444 (2014) 226-231.

[25] D. Philip, C. Unni, Extracellular biosynthesis of gold and silver nanoparticles using Krishna tulsi (Ocimum sanctum) leaf, Physica E Low Dimens. Syst Nanostruct. 43 (2011) 1318-1322.

[26] S. Pattanayak, M.M.R. Mollick, D. Maity, S. Chakraborty, S.K. Dash, et al., Butea monosperma bark extract mediated green synthesis of silver nanoparticles: Characterization and biomedical applications, J. Saudi Chem. Soc. 21 (2017) 673-684.

[27] C.S. Madhu, K.S. Balaji, A.C. Sharada, J. Shankar, Anticancer effect of silver nanoparticles (AgNP's) from Decalepis hamiltonii: An in vivo approach, Mater. Today Proc. 4 (2017) 11947-11958.

[28] B. Ajitha, Y. Ashok Kumar Reddy, H.J. Jeon, C.W. Ahn, Synthesis of silver nanoparticles in an eco-friendly way using Phyllanthus amarus leaf extract: Antimicrobial and catalytic activity, Adv. Powder Technol. 29 (2018) 86-93.

[29] K. Anandalakshmi, J. Venugobal, V. Ramasamy, Characterization of silver nanoparticles by green synthesis method using Pedalium murex leaf extract and their antibacterial activity, Appl. Nanosci. 6 (2016) 399-408.

[30] M.J. Masarudin, S.M. Cutts, B.J. Evison, D.R. Phillips, P.J. Pigram, Factors determining the stability, size distribution, and cellular accumulation of small, monodisperse chitosan nanoparticles as candidate vectors for anticancer drug delivery: application to the passive encapsulation of [(14)C]-doxorubicin Nanotechnol. Sci. Appl. 8 (2015) 67-80.

[31] S. Ramanathan, S.C.B. Gopinath, P. Anbu, T. Lakshmipriya, F.H. Kasim, C.G. Lee, Eco-friendly synthesis of Solanum trilobatum extract-capped silver nanoparticles is compatible with good antimicrobial activities, J. Mol. Struct. 1160 (2018) 80-91.

[32] S. Gurunathan, J.W. Han, D.N. Kwon, J.H. Kim, Enhanced antibacterial and antibiofilm activities of silver nanoparticles against Gram-negative and Grampositive bacteria, Nanoscale Res. Lett. 9(373) (2014) 1-17. 